\title{
Correção da Incontinência Urinária de Esforço com Sling: Resultados Iniciais
}

\author{
Stress Urinary Incontinence Correction with Sling: First Results \\ José Antônio M. Martins, Rodrigo de A. Castro, Manoel J. B. C. Girão \\ Marair G. F. Sartori, Edmundo C. Baracat, Geraldo R. de Lima
}

\section{RESUMO}

Objetivo: analisar os resultados cirúrgicos após slings com mucosa vaginal, realizados pelo setor de Uroginecologia e Cirurgia Vaginal da UNIFESP/EPM, no tratamento de mulheres incontinentes com hipermobilidade do colo vesical, que apresentam alto risco de falha cirúrgica para outras técnicas ou naquelas com defeito esfincteriano intrínseco e, ainda, recidivas cirúrgicas.

Métodos: foram avaliadas 21 pacientes submetidas à cirurgia para correção de incontinência urinária pela técnica de sling vaginal, no periodo de dezembro de 1997 a fevereiro de 1999, com seguimento pós-operatório que variou de 1 a 14 meses (média de 8,2). A média de idade das pacientes foi de 56 anos (39 a 77 anos), sendo que 15 (71,4\%) encontravam-se na menopausa e $6(28,6 \%)$ no menacme. Todas as pacientes foram avaliadas antes da cirurgia por meio de anamnese, exame clinico, estudo ultra-sonográfico e urodinâmico, sendo o grau de perda urinária acentuado em $66,7 \%$ e moderado em $33,3 \%$ das pacientes. Todas as pacientes apresentavam hipermobilidade da junção uretrovesical (superior a $10 \mathrm{~mm}$ ) e 12 pacientes apresentavam cirurgia prévia para correção de incontinência urinária. Ao estudo urodinâmico, as pacientes apresentavam perda urinária com pressão máxima de fechamento

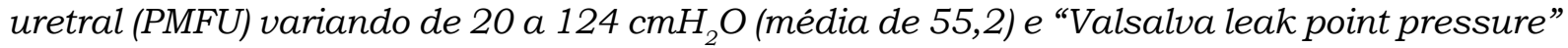
(VLPP) variando de 18 a $128 \mathrm{cmH}_{2} \mathrm{O}$ (média de 60,3). As indicações das cirurgias foram: defeito esfincteriano (11 pacientes - 52,4\%), obesidade (5 pacientes - 23,8\%), defeito esfincteriano o obesidade (2 pacientes - 9,5\%), recidiva cirúrgica (2 pacientes -9,5\%) e defeito esfincteriano e prolapso uterino de $1^{\circ}$ grau (1 paciente - 4,8\%).

Resultados: como complicações, 6 pacientes $(28,6 \%)$ apresentaram retenção urinária temporária no pós-operatório, 1 (4,8\%) infecção do trato urinário, $1(4,8 \%)$ presença de fio de polipropileno na vagina, 1 (4,8\%) infecção da ferida cirúrgica, 4 pacientes (19\%) evoluíram com urgência/incontinência, 1 (4,8\%) com urgência miccional e 1 (4,8\%) com dificuldade para urinar (elevado resíduo pós-miccional). O grau de satisfação das pacientes foi satisfatório, com 15 pacientes $(71,4 \%)$ referindo cura, 3 (14,3\%) melhora, 2 (9,5\%) quadro de perda urinária inalterado e 1 (4,8\%) piora da perda urinária.

Conclusões: a cirurgia de sling com mucosa vaginal é eficaz para o tratamento de casos específicos de incontinência urinária de esforço, destacando-se defeito esfincteriano, recidivas cirúrgicas e fatores predisponentes para falha de outras técnicas.

PALAVRAS-CHAVE: Incontinência urinária. Incontinência urinária: tratamento cirúrgico. Cirurgia: complicações. Estudo urodinâmico.

Disciplina de Ginecologia, Departamento de Tocoginecologia, Universidade Federal de São Paulo/Escola Paulista de Medicina

Correspondência:

José Antônio Morais Martins

Rua Borges Lagoa, 933, apto. 84 - Vila Clementino

04038-032 - São Paulo - SP

\section{Introdução}

A cirurgia de sling foi introduzida em 1907 por Giordano, utilizando uma dobra do músculo grácil. Posteriormente, inúmeras modificações na 
técnica surgiram ${ }^{1}$. É eficaz para o tratamento da incontinência urinária de esforço (IUE) devida tanto à hipermobilidade uretral quanto ao defeito esfincteriano intrínseco ${ }^{2}$.

As diferentes técnicas são similares, diferindo apenas em três pontos básicos: tipo de material utilizado, que pode ser orgânico (autólogo ou heterólogo) ou sintético, vias de acesso (abdominal ou abdominal e vaginal) e pontos de fixação. $\mathrm{O}$ material ideal para a realização do sling deve promover adequada força de tensão, ser facilmente acessivel e ter baixo risco de infecção, rejeição e cicatrização excessiva ${ }^{1}$.

Em particular, a técnica que utiliza um retalho da mucosa vaginal suburetral funciona elevando esse retalho ao nível da junção uretrovesical, criando, assim, um ligamento pubouretral artificial. Em conseqüência, estabiliza a junção uretrovesical e dá um novo suporte para a uretra, facilitando, desta maneira, o funcionamento do seu mecanismo intrínseco $0^{1,3,4}$.

É indicada principalmente para IUE recidivada, para os casos de defeito esfincteriano (em especial aqueles com hipermobilidade do colo vesical) e para pacientes com alto risco de falha cirúrgica (bronquite crônica, asma, flacidez congênita dos tecidos, atividades recreacionais ou profissionais de levantamento de peso ou alto impacto, obesidade grave e uso crônico de esteróide). É contra-indicada em pacientes com bexiga flácida ou sem reflexos, com elevado resíduo pósmiccional, bexiga neurogênica, instabilidade grave do detrusor, refluxo vesicoureteral, história de irradiação pélvica, fisstula vésico ou uretrovaginal, desejo futuro de gravidez e indisposição ou inabilidade da paciente de realizar autocateterização em casos de retenção urinária pós-operatória ${ }^{1}$.

As taxas de cura após cirurgia de sling variam, na literatura, de 70 a $100 \%$, variação esta que está influenciada por diversos fatores, como modificações da técnica, experiência do cirurgião, diversidade de pacientes e critérios subjetivos de cura, que dificultam a comparação entre os autores $^{5}$.

Historicamente, a cirurgia de sling dá bons resultados, mas apresenta altas taxas de retenção urinária prolongada e instabilidade do detrusor secundária. No entanto, proporciona alto grau de satisfação às pacientes, a despeito de eventuais desconfortos no pós-operatório ${ }^{6}$.

A literatura sugere maiores taxas de complicações quando materiais sintéticos são utiliza$\operatorname{dos}^{7}$.

As vantagens do sling com mucosa vaginal em relação às demais técnicas são: a simplicidade, necessidade de apenas pequena incisão abdominal, menor tempo operatório, utilização de um tecido in situ (bem vascularizado e saudável), além de menor tempo de internação ${ }^{8}$.

O objetivo deste trabalho foi analisar os resultados cirúrgicos após slings com mucosa vaginal, realizados pelo setor de Uroginecologia e Cirurgia Vaginal, no tratamento de mulheres incontinentes com hipermobilidade do colo vesical, que apresentam alto risco de falha cirúrgica para outras técnicas, ou naquelas com defeito esfincteriano intrínseco e, ainda, recidivas cirúrgicas.

\section{Pacientes e Métodos}

Foram avaliadas 21 pacientes submetidas à cirurgia para correção de incontinência urinária pela técnica de sling vaginal, no período de dezembro de 1997 a fevereiro de 1999, com seguimento pós-operatório que variou de um a quatorze meses (média de 8,2).

A média de idade das pacientes foi de 56 anos (38 a 77 anos). Das pacientes, 15 (71,4\%) encontravam-se na menopausa e $6(28,6 \%)$ no menacme.

Todas as pacientes foram avaliadas antes da cirurgia por meio de anamnese, exame clinico, estudo ultra-sonográfico e urodinâmico (fluxometria, cistometria e perfil uretral).

O estudo ultra-sonográfico foi realizado nas pacientes com bexiga cheia, em posição ginecológica, localizando-se, com o transdutor supraclitoridiano, a uretra, a bexiga e a junção uretrovesical. As pacientes eram solicitadas a realizar manobra de Valsalva e a mobilidade da junção uretrovesical era medida. Uma mobilidade superior a $10 \mathrm{~mm}$ era considerada hipermobilidade. Observava-se, também, se ocorria perda urinária.

A fluxometria foi realizada utilizando-se um fluxômetro com sensores de peso, e os principais parâmetros avaliados e seus respectivos padrões de normalidade foram o padrão da curva (bem definida), o fluxo máximo ( $>15 \mathrm{ml} / \mathrm{s}$ ), o tempo de micção $(<20$ s), tempo para atingir o fluxo máximo $(<10$ $\mathrm{s})$, resíduo vesical $(<50 \mathrm{ml})$ e volume eliminado.

A cistometria foi realizada utilizando-se sonda uretral número 8 para infusão de água destilada a uma velocidade da 100 gotas/min, balão retal para medida da pressão intra-abdominal e cateter vesical para medida da pressão intravesical. Os principais parâmetros avaliados foram o primeiro desejo miccional, a capacidade vesical máxima, perda urinária, contrações não-inibidas e pressões de perda ("Valsalva leak point pressure" - VLPP) com $200 \mathrm{ml}$. Valores da VLPP inferiores a $60 \mathrm{~cm} /$ $\mathrm{H}_{2} \mathrm{O}$ foram considerados sugestivos de defeito esfincteriano. 
O perfil uretral foi realizado utilizando-se cateter de fluxo número 8 e os parâmetros avaliados foram a pressão máxima de fechamento uretral (PMFU) e o comprimento funcional da uretra. Valores da PMFU inferiores a $20 \mathrm{~cm} / \mathrm{H}_{2} \mathrm{O}$ foram considerados sugestivos de defeito esfincteriano.

O grau de perda urinária referido pelas pacientes era acentuado em $66,7 \%$ e moderado em $33,33 \%$ das pacientes.

Ao estudo ultra-sonográfico do colo vesical, $100 \%$ das pacientes apresentavam hipermobilidade da junção uretrovesical (acima de $10 \mathrm{~mm}$ ).

Ao estudo urodinâmico, as pacientes apresentavam perda urinária com PMFU variando de 20 a $124 \mathrm{cmH}_{2} \mathrm{O}$ (média de 55,2) e VLPP variando de 18 a $128 \mathrm{cmH}_{2} \mathrm{O}$ (média de 60,3).

Das 21 pacientes, $12(57,1 \%)$ apresentavam cirurgia prévia para correção de incontinência urinária, sendo que 4 delas haviam sido submetidas a Kelly-Kennedy, 2 a Burch, 4 a Kelly-Kennedy e Burch, 1 a Burch e Gittes e 1 a Burch e injeção peri-uretral (Tabela 1).

Tabela 1 - Pacientes com cirurgias prévias (12/21) para correção de incontinência urinária.

\begin{tabular}{lcc}
\hline Cirurgias & $\mathbf{n}$ & $\%$ \\
\hline Kelly-Kennedy & 4 & 33,3 \\
Burch & 2 & 16,7 \\
Kelly-Kennedy/Burch & 4 & 33,3 \\
Burch/Gittes & 1 & 8,3 \\
Burch/injeção periuretral & 1 & 8,3 \\
Total & 12 & 100 \\
\hline
\end{tabular}

As indicações da cirurgias de sling foram: defeito esfincteriano (11 pacientes $-52,4 \%$ ), obesidade $(5-23,8 \%)$, defeito esfincteriano e obesidade $(2-9,5 \%)$, defeito esfincteriano e prolapso uterino de I grau $(1-4,8 \%)$ e recidiva cirúrgica $(2$ $-9,5 \%)$ (Tabela 2).

Tabela 2 - Indicações das cirurgias.

\begin{tabular}{lrr}
\hline Indicações & $\mathbf{n}$ & \% \\
\hline Defeito esfincteriano & 11 & 52,4 \\
Obesidade & 5 & 23,8 \\
Defeito esfincteriano/obesidade & 2 & 9,5 \\
Defeito esfincteriano/prolapso uterino de I grau & 1 & 4,8 \\
Recidiva pós-Burch & 2 & 9,5 \\
Total & 21 & 100 \\
\hline
\end{tabular}

A técnica da cirurgia de sling com mucosa vaginal consiste na dissecção de um retalho de mucosa vaginal ao nivel da junção uretrovesical no tamanho de $2 \mathrm{~cm}$ por $2 \mathrm{~cm}$, no qual são fixados pontos de Prolene ${ }^{\circledR} 0$ nos limites laterais superiores e inferiores. Faz-se uma dissecção mais lateral ao retalho da mucosa vaginal e perfura-se a fáscia endopélvica com uma pinça de Kelly angulada 45 graus em relação ao plano horizontal, em direção ao ombro da paciente. Procede-se então a uma dissecção digital do espaço de Hetzius rente à face posterior da sinfise púbica até a aponeurose do músculo reto abdominal. Faz-se uma incisão na pele e no tecido celular subcutâneo até exposição da aponeurose do reto abdominal. Com a utilização de uma agulha de Stamay, perfura-se essa aponeurose guiando-se pelo indicador via vaginal até exteriorização pela vagina, procedendo-se então à elevação dos fios de Prolene ${ }^{\circledR}$ até a aponeurose do reto abdominal, sobre a qual serão dados pontos unindo os fios de Prolene ${ }^{\circledR}$ anteriores e posteriores, visualizandose, pela cistoscopia, a elevação e fechamento do colo vesical. Ao final realiza-se a cistoscopia e o fechamento da pele e da mucosa vaginal adjacente ao retalho no sentido ântero-posterior, embutindo-o.

\section{Resultados}

Das 21 pacientes submetidas à cirurgia, 15 $(71,4 \%)$ apresentaram-se continentes, $3(14,3 \%)$ com melhora, $2(9,5 \%)$ com quadro de perda urinária inalterado e $1(4,8 \%)$ paciente com piora da perda urinária.

Como complicações, 6 pacientes $(28,6 \%)$ apresentaram retenção urinária temporária no pós-operatório, $1(4,8 \%)$ infecção do trato urinário, $1(4,8 \%)$ presença de fio de Prolene ${ }^{\circledR}$ na vagina e 1 $(4,8 \%)$ infecção da ferida cirúrgica. Não houve casos de lesão uretral ou vesical, dispareunia, fistula uretro ou vesicovaginal, necrose uretral, erosão ou remoção do sling, porém 4 pacientes (19\%) evoluíram com urgência/incontinência, 1 (4,8\%) com urgência miccional e $1(4,8 \%)$ com dificuldade para urinar (elevado resíduo pós-miccional (Tabela 3).

\section{Discussão}

A incontinência urinária de esforço (verdadeira) é a causa mais freqüente de perda urinária e apresenta mecanismo fisiopatológico multifatorial. Como conseqüência, apresenta diversas formas de tratamento. 
Tabela 3 - Complicações do sling vaginal

\begin{tabular}{lcc}
\hline Complicações & $\mathbf{n}$ & $\%$ \\
\hline Retenção urinária temporária & 6 & 40 \\
Infecção do trato urinário & 1 & 6,6 \\
Presença de fio de Prolene® na vagina & 1 & 6,6 \\
Infecção da ferida cirúrgica & 1 & 6,6 \\
Urgência/incontinência & 4 & 26,7 \\
Urgência miccional & 1 & 6,6 \\
Dificuldade para urinar & 1 & 6,6 \\
(elevado resíduo pós-miccional) & & \\
Lesão uretral & - & - \\
Dispareunia & - & - \\
Fistula uretro ou vesicovaginal & - & - \\
Necrose uretral & - & - \\
Erosão ou remoção do "sling" & - & - \\
Total & 15 (71,4\%) & 100 \\
\hline
\end{tabular}

A cirurgia para correção de IUE é eletiva. É uma decisão de qualidade de vida. Portanto, a decisão de operar deve ser da paciente e após um diagnóstico claramente estabelecido, sendo as chances de sucesso muito maiores quando a paciente entende o procedimento com suas possíveis complicações e quando tem a oportunidade de considerar outras formas de terapia, tentandoas ou rejeitando-as ${ }^{9}$.

A cirurgia de sling, quando indicada, deve estabilizar e suportar, sem elevar exageradamente e obstruir a junção uretrovesical, tendo como finalidade a restauração da normalidade anatômica e funcional da bexiga ${ }^{9}$. A cirurgia de sling com mucosa vaginal apresenta as vantagens de ser facilmente acessivel, ter baixo risco de infecção, rejeição e cicatrização excessiva, visto que é um tecido in situ, bem vascularizado e saudável ${ }^{8}$. Tecidos sintéticos tendem a apresentar taxas de complicações maiores quando são usados para o sling ${ }^{7}$.

Uma seleção cuidadosa das pacientes aumenta o sucesso cirúrgico e minimiza as complicações pós-operatórias.

Em nosso levantamento destacam-se: $74 \%$ das pacientes submetidas à cirurgia de sling vaginal encontravam-se na pós-menopausa, com idade média de 56 anos, $57,1 \%$ das pacientes já tinham sido submetidas a cirurgias prévias para correção de IUE e $52,4 \%$ delas tinham como indicação o defeito esfincteriano.

Isso evidencia ser a cirurgia de sling uma opção terapêutica para casos de dificil tratamento, com múltiplos fatores etiológicos para IUE, em pacientes muitas vezes com IUE recidivadas, devido, não raro, a diagnósticos não muito bem es- clarecidos.

Evidencia, também, como principal indicação para o sling vaginal o defeito esfincteriano intrinseco, em especial com hipermobilidade do colo vesical. Portanto, é imperativo identificar essa alteração antes de qualquer medida terapêutica para correção de IUE.

Juma et al. ${ }^{10}$, avaliando 54 pacientes com defeito esfincteriano submetidas a sling vaginal, obtiveram uma taxa de cura de $94,4 \%$ num seguimento médio de 23,9 meses, com poucas complicações pós-operatórias, resultado superior ao por nós analisado $(71,43 \%$ de cura), porém $83 \%$ das pacientes apresentaram retenção urinária temporária, com 5,5\% delas necessitando de autocateterização intermitente, ao passo que a retenção urinária temporária por nós observada foi de $28,6 \%$. Tais diferenças podem ser explicadas, em parte, pela dificuldade de padronização no ajuste da tensão das suturas de sustentação do sling, mas caracterizam a cirurgia de sling como retencionista.

Haab et al. ${ }^{6}$, analisaram, mediante questionário, 37 pacientes com defeito esfincteriano submetidas à cirurgia de sling vaginal. Destas, 86\% relataram melhora da incontinência urinária e $81 \%$ recomendariam a cirurgia. Tais dados se assemelham aos por nós analisados $(71,4 \%$ de cura e $14,3 \%$ de melhora) e confirmam a grande satisfação das pacientes, apesar de significantes sintomas pós-operatórios como retenção urinária e urgência miccional, esta provavelmente decorrente de fatores irritativos locais.

O grau de satisfação das pacientes por nós analisado também foi semelhante ao relatado por Raz et al. ${ }^{8}$ que, analisando 26 pacientes sem incompetência uretral neurogênica, submetidas à cirurgia de sling vaginal, observaram resultados excelentes em 20 pacientes $(77 \%)$, muito bons em 2 (8\%), melhora em 1 (4\%) e falha em $3(12 \%)$.

Podemos concluir, portanto, que a cirurgia de sling com mucosa vaginal é eficaz para o tratamento de casos específicos de IUE, destacando-se defeito esfincteriano, recidivas cirúrgicas e fatores predisponentes para falha de outras técnicas.

\section{SUMMARY}

Purpose: to analyze the surgical results after slings with vaginal wall, performed by the Urogynecology and Vaginal Surgery Sector of UNIFESP/EPM, for the treatment of incontinent women with hypermobility of the bladder neck, who show great risk of surgery failure with other techniques 
or in those with intrinsic sphincteric deficiency (ISD) and, also, surgery recurrence.

Methods: we studied 21 patients submitted to surgery in order to correct urinary incontinence by the vaginal wall sling technique, in the period from December 1997 to February 1999, with postoperative follow-up which varied between 1 and 14 months (average 8.2). The mean age of patients was 56 years (39 to 77 years), 15 (71.4\%) were in menopause and $6(28.6 \%)$ in menacme. All patients were evaluated before the surgery through medical interview, physical examination, ultrasound and urodynamic study, the grade of urinary loss being high in $66.7 \%$ and moderate in $33.3 \%$ of the patients. All patients showed hypermobility of the bladder neck (more than $10 \mathrm{~mm}$ ) and 12 patients had previous surgery to correct the urinary incontinence. Regarding the urodinamic study, the patients manifested urinary loss with maximum pressure of urethral closure (MPUC) varying from 20 to $124 \mathrm{cmH}_{2} \mathrm{O}$ (average 55.2) and Valsalva leak point pressure (VLPP) varying from 18 to 128 $\mathrm{cmH}_{2} \mathrm{O}$ (average 60.3). The indications of surgery were: ISD (11 patients $-52.4 \%)$, obesity (5 patients $-23.8 \%$ ), ISD and obesity (2 patients - 9.5\%), surgery recurrence (2 patients 9.5) and ISD and first grade womb prolapse (1 patient $4.8 \%$ ).

Results: as complications, 6 patients (28.6\%) showed temporary urinary retention after surgery, 1 patient (4.8\%) infection in the urinary tract, 1 patient (4.8\%) presence of polypropylene suture in the vagina, 1 patient (4.8\%) infection of the surgery wound, 4 patients (19\%) developed urgency/ incontinence, 1 (4.8\%) urgency and 1 (4.8\%) difficulty in urinating (high postvoiding residue). The grade of the patients' satisfaction was satisfactory, with 15 patients (71.4\%) referring cure, 3 patients (14.3\%) improvement, in 2 patients (9.5\%) the urinary loss remained unchanged and in 1 patient (4.8\%) the urinary loss got worse.

Conclusions: the vaginal wall sling surgery is efficient for the treatment of specific cases of stress urinary incontinence, emphasizing intrinsic urethral sphincteric incompetence, surgery recurrence and predisposing factors to failure of other techniques.

KEY WORDS: Female urinary incontinence. Sling surgery. Urinary incontinence: surgical treatment. Urodynamic evaluation.

\section{Referências}

1. Horback NS. Suburethral sling procedures. In: Ostergard DR, Bent AE, editores. Urogynecology and Urodynamics: theory and practice. $4^{\text {th }}$ ed. Baltimore: Williams \& Wilkins; 1996. p.569-79.

2. Rovner ES, Ginsberg DA, Raz S. The UCLA surgical approach to sphincteric incontinence in women. World J Urol 1997; 15:280-94.

3. Cespedes RD, Cross CA, McGuire EJ. Pubovaginal fascial slings. Tech Urol 1997; 3: 195-201.

4. Girão MJBC, Baracat EC, Lima GR. Tratamento cirúrgico. In: Girão MJBC, Baracat EC, Lima GR, editores. Uroginecologia. $1^{a}$ ed. São Paulo: Artes Médicas; 1997. p.46.

5. Mclennan MT, Bent AE, Richardson DA. Evaluation of different surgical procedures. In: Ostergard DR, Bent AE, editores. Urogynecology and Urodynamics: theory and practice. $4^{\text {th }}$ ed. Baltimore: Williams \& Wilkins; 1996. p.517-26.

6. Haab F, Trockman BA, Zimmern PE, Leach GE. Results of pubovaginal sling for the treatment of intrinsic sphincteric deficiency determined by questionnaire analysis. J Urol 1997; 158:173841.

7. Leach GE, Dmochowski RR, Appell RA, et al. Female Stress Urinary Incontinence Clinical Guidelines Panel summary report on surgical management of female stress urinary incontinence. The American Urological Association. J Urol 1997; 158:875-80.

8. Raz S, Siegel AL, Short JL, Snyder JA. Vaginal wall sling. J Urol 1989; 141:43-6.

9. Wall LL, Norton PA. Surgical management of stress incontinence. In: Wall LL, Norton PA, editores. Practical Urogynecology. $1^{\text {st }}$ ed. Baltimore: Williams \& Wilkins; 1993. p.153-90.

10. Juma S, Little NA, Raz S. Vaginal wall sling: four years later. Urology 1992; 39:424-8. 